\title{
Experimental demonstration of adiabatic coupling into SOI photonic crystal coupled-cavity waveguides
}

\author{
P. Sanchis, A. Martínez, J. García, F. Cuesta-Soto and J. Martí \\ Valencia Nanophotonics Technology Center, Universidad Politécnica de Valencia, Camino de Vera s/n, 46022, Valencia, Spain \\ E-mail: pabsanki@ntc.upv.es \\ P. Dumon, W. Bogaerts, D. Van Thourhout and R. Baets \\ Ghent University-IMEC, Department of Information Technology, Sint-Pietersnieuwstraat 41, B-9000 Ghent, Belgium
}

\begin{abstract}
The experimental demonstration of an adiabatic coupling technique between Siliconon-insulator photonic crystal single-line defect waveguides and coupled-cavity waveguides is reported. Experimental results are compared with three dimensional finite-difference time-domain simulation results showing very good agreement.

(C) 2005 Optical Society of America
\end{abstract}

OCIS codes: (230.7370) Waveguides; (130.3120) Integrated optics devices; (250.5300) Photonic integrated circuits

\section{Introduction}

Photonic crystal $(\mathrm{PhC})$ coupled-cavity waveguides (CCWs) may be a good choice to implement different optical functionalities, such as very compact optical delay lines or dispersion compensators, due to their inherent very small group velocity [1]. However, one key issue that needs to be addressed is an efficient coupling from an external medium. An adiabatic coupling technique between single-line-defect waveguides (SLWGs) and CCWs was recently proposed [2]. Furthermore, their dynamic performance by analyzing the transmission of ultrashort pulses through the structure was also investigated [3]. The coupling technique is based on gradually varying the radii of the spacing defects between cavities, as it can be seen in Fig. 1(a). In this paper, the experimental demonstration of this coupling technique by using an air-hole PhC structure fabricated in Silicon-on-insulator (SOI) is reported.
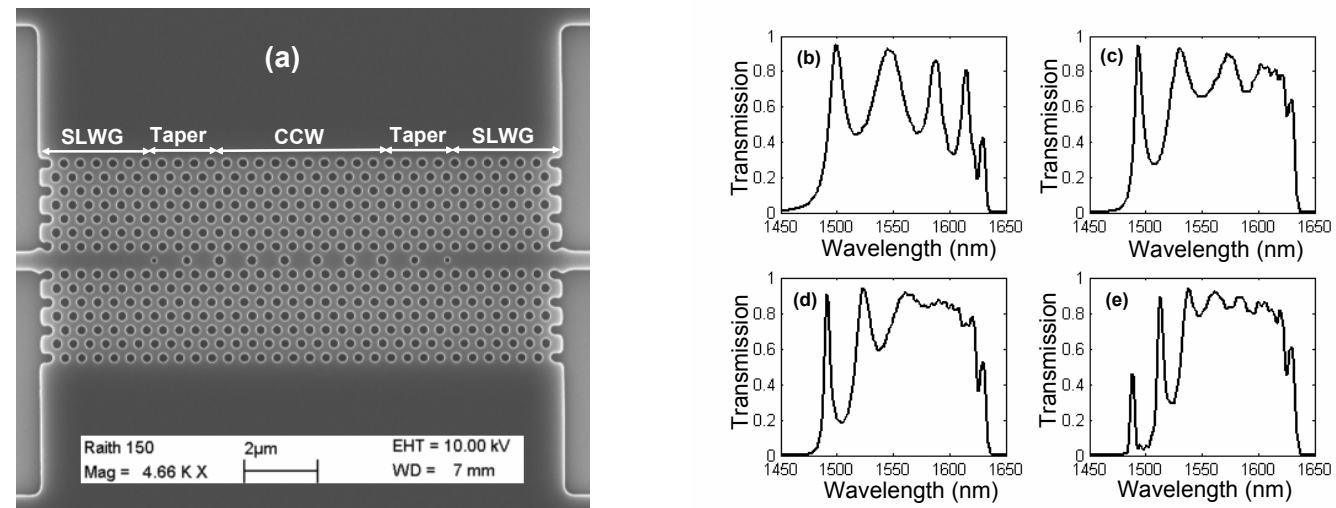

Fig. 1.- (a) Scanning electronic microscope (SEM) image of the coupled cavity waveguide (CCW) coupled to the singleline defect waveguides (SLWGs) with the adiabatic taper. The taper is based on gradually varying the radii of the spacing holes between cavities. The 2D simulated transmission spectra as a function of the wavelength for a CCW length of 5 cavities and for different taper lengths is shown in the right part for: (b) without taper, (c) $\mathrm{L}=2$, (d) $\mathrm{L}=3$ and (e) $\mathrm{L}=5$. The parameter $L$ refers to the number of intermediate holes, with a lineal variation of their radius, used in the taper.

\section{Simulation results}

The bulk $\mathrm{PhC}$ is a two-dimensional (2D) triangular array of air holes in a dielectric background of Silicon. 2D finitedifference time-domain (FDTD) simulations have been initially considered for the analysis. The effective index approximation $\left(\mathrm{n}_{\mathrm{eff}}=2.8\right)$ is used for the vertical direction and the hole radius is of $R=0.26 a, a$ being the lattice constant. The effective index was calculated for a thickness of the Silicon layer of $220 \mathrm{~nm}$ in agreement with the fabricated structure. A Silicon waveguide with a cladding of air is used for coupling into and out of the SLWGs. The thickness of the waveguide $(w=0.6 \mu \mathrm{m})$ has been chosen to achieve negligible coupling losses into the SLWG. The transmission spectra as a function of the wavelength for a CCW length of 5 cavities and for different taper lengths are shown in Fig. 1(b)-(e). A lattice constant of $a=445 \mathrm{~nm}$ has been used in agreement with that employed in the fabricated structure. When no taper is used, a number of resonances peaks equal to the number of cavities appear in 


\section{CTuDD2}

the transmission spectrum, as it can be seen in Fig. 1(a). The spacing between adjacent resonance peaks is smaller at higher wavelengths because the dispersion relation of the $\mathrm{CCW}$ is flatter in this region, which is more suitable for implementing functionalities based on the high dispersion of the CCW. Therefore, the elimination of the resonance peaks is desired, especially if ultrashort pulses are transmitted through the structure. The undesired resonance peaks are eliminated by the introduction of the adiabatic taper in the structure. The underlying physics of the taper is an adiabatic matching of the dispersion relations of the SLWG and the CCW [3].

\section{Experimental results}

The samples were fabricated on an 8" SOI wafer using 248-nm deep UV lithography [4]. The SOI wafer consists of a $220 \mathrm{~nm}$-thick Silicon layer, where the air holes lattice is etched, placed on top of a $1 \mu \mathrm{m}$-thick silica layer deposited over a bare Silicon substrate. The transmission as a function of wavelength was measured using an end-fire technique. Light from a tunable laser source was coupled into a $3 \mu \mathrm{m}$-wide ridge waveguide using a lensed fiber. The output power of the laser was $1 \mathrm{~mW}(0 \mathrm{dBm})$. The samples consist of $3 \mu \mathrm{m}$-wide ridge waveguides tapered down to a $500 \mathrm{~nm}$-wide waveguides, best suited for coupling light into and out of the PhC. The output light was collected by an objective onto a power detector. A polarizer selecting the TE polarization and a diaphragm to collect only the output light from the ridge waveguide were used before the power detector.
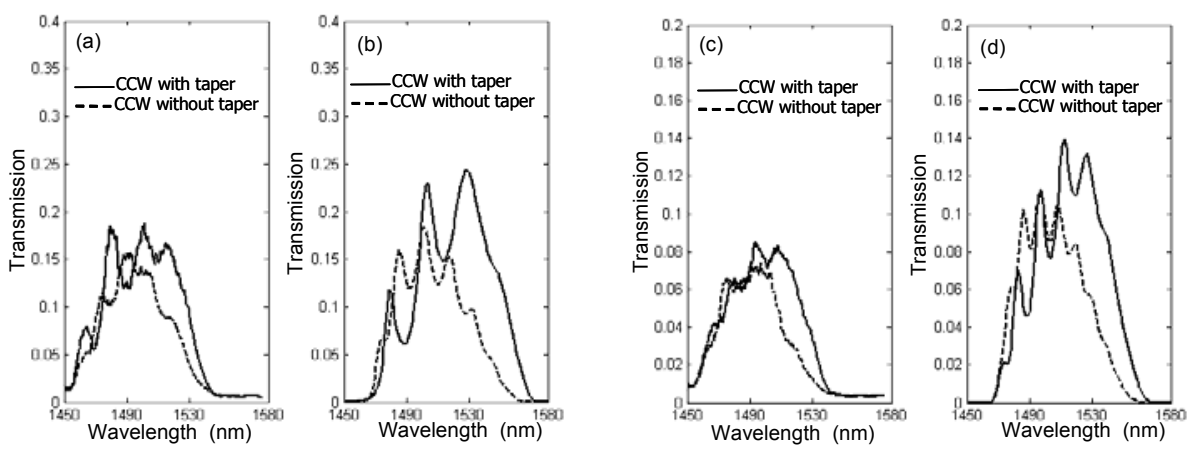

Fig. 2. (a) Experimental and (b) 3D FDTD simulated transmission spectra as a function of the wavelength for a CCW of 10 cavities and a taper/CCW/taper structure of 9 cavities. (c) Experimental and (d) 3D FDTD simulated transmission spectra as a function of the wavelength for a $\mathrm{CCW}$ of 15 cavities and a taper/CCW/taper structure of 14 cavities.

CCWs of different lengths coupled to the SLWGs with and without taper were fabricated. Experimental results have been compared with 3D FDTD simulation results for a more detailed and rigorous analysis. Fig. 2(a)-(b) shows the transmission efficiency as a function of the wavelength for a CCW of 10 cavities and a taper/CCW/taper structure of 9 cavities while Fig. 2(c)-(d) shows the transmission spectra for a CCW of 15 cavities and a taper/CCW/taper structure of 14 cavities. Experimental results have been normalized by the averaged transmission spectrum of the unpatterned structure. It can be seen that a very good agreement is achieved between experimental and 3D FDTD results. The transmitted power is improved at higher wavelengths when the adiabatic taper, of only two intermediate holes, is used to couple light into and out the CCW. This transmission improvement is in agreement with the one obtained by the 2D FDTD simulation results. However, although the lower band edge of the transmission spectra is also in agreement between experimental and 2D simulation results, the bandwidth is reduced for the experimental results, which restricts the transmission improvement provided by the taper. Furthermore, transmission losses are rather high in both the CCW with and without taper structures.

Transmission losses are due to the propagation losses of the CCW and the SLWG and the coupling losses between the dielectric waveguide and the SLWG and between the SLWG and the CCW. The only difference between the results shown in Fig. 2(a) and Fig. 2(c) is the different length of the CCW since the taper length is not modified. Therefore, the transmission efficiency is only decreased due to the propagation losses in the CCW. Concretely, the transmitted power is decreased around $3 \mathrm{~dB}$ when the $\mathrm{CCW}$ length is increased by $4.45 \mu \mathrm{m}(10 a)$ so the propagation losses of the $\mathrm{CCW}$ are roughly estimated at $0.67 \mathrm{~dB} / \mu \mathrm{m}$. These rather high propagation losses are attributed due to the out-of-plane losses originated from the fact that the CCW operates above the light line, which implies that the guided mode is intrinsically lossy. It is important to remark that propagation losses due to sidewall roughness are not taken into account in the simulations.

The transmission losses due to the estimated propagation losses are of $6.3 \mathrm{~dB}(23 \%)$ for a CCW of 10 cavities and of $9.3 \mathrm{~dB}(11.75 \%)$ for a CCW of 15 cavities. These efficiencies are close to those obtained for the CCW without taper, shown with dashed lines in Fig. 2(a) and Fig. 2(c), which means that the propagation losses of the CCW are clearly 


\section{CTuDD2}

dominant in the overall transmission losses. The transmission efficiency is improved for the CCW with taper structure. However, these lower transmission losses are not only due to the higher coupling efficiency between the SLWG and CCW but also because the propagation losses in the taper are lower than the ones given for the CCW. It is important to notice that the number of cavities is almost the same in both the CCW with and without taper structures so the $\mathrm{CCW}$ length is actually shorter in the former.

In order to better evaluate the taper performance, the transmission spectra for a CCW of the same length coupled to the SLWG with and without the adiabatic taper have been obtained by means of 3D FDTD simulations. Furthermore, the total length in both structures has also been fixed to be the same. Fig. 3(a) shows the transmission spectra considering a CCW of 5 cavities. The transmission spectrum is also shown for a SLWG with a length equal to the sum of the lengths of the input and output SLWGs used in the CCW with taper structure. On the other hand, Fig.3(b) shows the transmission spectra for the $\mathrm{CCW}$ with and without taper structures calculated by means of 2D FDTD simulations. First of all, it can be seen that the transmitted power in the SLWG decreases as the wavelength increases when the wavelength is approximately higher than $1530 \mathrm{~nm}$. This power drop together with the high propagation losses suffered in the CCW originates that the bandwidth of the CCW is reduced with respect to the broader bandwidth predicted from the $2 \mathrm{D}$ simulations. Therefore, it is difficult to evaluate the taper performance as the transmission improvement occurs mainly at the higher wavelengths. However, it can still be seen that the transmission for the CCW with taper gets worse at lower wavelengths but it is improved at high wavelengths due to the effect of the taper, in agreement with the results predicted by the 2D results shown in Fig. 3(b), which demonstrates the performance improvement when the proposed adiabatic taper is used for coupling light into and out of the CCW.
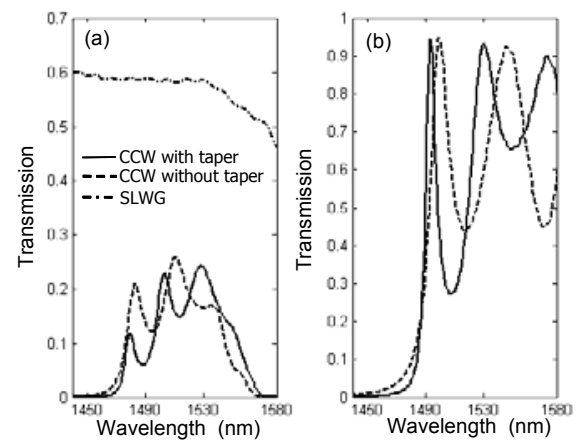

Fig. 3. (a) 3D and (b) 2D FDTD simulated transmission efficiency as a function of the wavelength. The transmission spectrum, calculated by means of 3D FDTD simulations, is also shown for a SLWG with a length equal to the sum of the lengths of the input and output SLWGs used in the CCW with taper structure.

\section{Conclusion}

Experimental results of an adiabatic coupling technique to improve the coupling efficiency into CCWs have been reported and compared with 2D and 3D FDTD simulation results. However, we have obtained that out-of-plane losses are rather high in CCW increasing significantly the transmission losses. Therefore, the analysis of out-ofplane losses and techniques to avoid them will become necessary to improve the CCW performance.

\section{References}

[1] A. Yariv, Y. Xu, R. K. Lee, and A. Scherer, "Coupled resonator optical waveguides: A proposal and analysis," Opt. Lett. 24, 711-713 (1999).

[2] P. Sanchis, J. Garcia, A. Martínez, F. Cuesta, A. Griol, and J. Martí, "Analysis of adiabatic coupling between photonic crystal single-line-defect and coupled-resonator optical waveguides," Opt. Lett. 28, 1903-1905 (2003).

[3] P. Sanchis, J. García, A. Martinez, J. Martí, "Pulse Propagation in Adiabatically Coupled Photonic Crystal Coupled Cavity Waveguides", to be published in J. Appl. Phys.

[4] W. Bogaerts, D. Taillaert, B. Luyssaert, P. Dumon, J. Van Campenhout, P. Bienstman, D. Van Thourhout, R. Baets, V. Wiaux, S. Beckx, "Basic structures for photonic integrated circuits in Silicon-on-insulator," Optics Express 12, 1583-1591 (2004). 